\title{
Effects of circulation and buoyancy on the transition from a fire whirl to a blue whirl
}

\author{
Sriram Bharath Hariharan* \\ Department of Mechanical Engineering, University of Maryland, College Park, Maryland 20742, USA
}

Yu Hu ${ }^{*} *$

Laboratory of Advanced Space Propulsion, Beijing Institute of Control Engineering, Beijing, People's Republic of China

\author{
Michael J. Gollner ${ }^{\dagger}$ \\ Department of Mechanical Engineering, University of California, Berkeley, Berkeley, California 94720, USA
}

Elaine S. Oran (1)

Department of Aerospace Engineering, Texas A\&M University, College Station, Texas 77843, USA

(Received 6 June 2020; accepted 9 September 2020; published 14 October 2020)

\begin{abstract}
The relative influence of circulation and buoyancy on fire whirls (FWs), blue whirls (BWs), and the transition between these regimes of a whirling flame is investigated using a combination of experimental data and scaling analyses. FWs are whirling, turbulent, cylindrical yellow (sooting) flame structures that form naturally in fires and are here created in laboratory experiments. In contrast, a BW is a laminar, blue flame (nonsooting) with an inverted conical shape. Measurements of the circulation and heat-release rate are combined with measurements of the flame geometry, defined by the flame width and the height, to provide characteristic length scales for these whirling-flame regimes. Using these, a nondimensional circulation $\left(\Gamma_{f}^{*}\right)$ and a heat-release rate $\left(\dot{Q}_{f}^{*}\right)$ were defined and shown to correspond to azimuthal and axial (buoyancy driven) momenta, respectively. The ratio $\mathcal{R}^{*}=\Gamma_{f}^{*} / \dot{Q}_{f}^{*}$, a quantity analogous to the swirl number used to characterize swirling jets, was evaluated for FWs and BWs. For FWs, $\mathcal{R}^{*}<1$, so that axial momentum is greater than azimuthal momentum and the flame is dominated by buoyant momentum. For BWs, $\mathcal{R}^{*}>1$, so that the flame is circulation dominated. This is argued to be consistent with vortex breakdown being an important part of the transition of FWs to BWs. This work presents a basis for predicting when a BW will form and remain a stable regime.
\end{abstract}

DOI: 10.1103/PhysRevFluids.5.103201

\section{INTRODUCTION}

The interaction of reacting buoyant plumes and swirling flow fields gives rise to structures called fire whirls. The formation and dynamics of fire whirls are important because of the devastating

\footnotetext{
${ }^{*}$ S.B.H. and Y.H. contributed equally to this article.

†mgollner@berkeley.edu

Published by the American Physical Society under the terms of the Creative Commons Attribution 4.0 International license. Further distribution of this work must maintain attribution to the author(s) and the published article's title, journal citation, and DOI.
} 


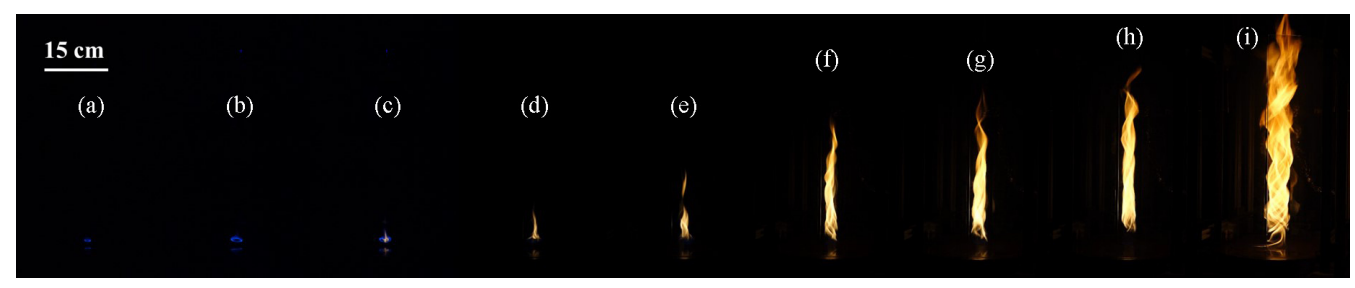

FIG. 1. Images of whirling-flame regimes formed at a gap size of $S=35 \mathrm{~mm}$. The experimental configuration is shown in Fig. 4. The fuel burning rate, $\dot{V}$, varies from panels (a) to (i) as $0.5,1.1,1.5,2,3,4.5,6,8$, and $10 \mathrm{ml} / \mathrm{min}$. Panels (a) and (b) show BWs, panels (c)-(e) show TBWs, and panels (f)-(i) show FWs.

effects they can have in urban and wildland fires [1-3]. Both the structure and the dynamic behavior of fire whirls are dominated by the effects of buoyancy, which arises from heat release, and by circulation, determined by the level of ambient swirl. In laboratory-scale experimental investigations, the combination of the heat-release rate $(\dot{Q})$ and circulation $(\Gamma)$ can be controlled to determine the shapes of fire whirls, which have been classified into a number of regimes [4].

The blue whirl is a small, soot-free flame that was first observed in an experimental study of fire whirls formed on a water surface [5]. The transition to the blue whirl occurred naturally, as air was entrained through tangential inlets and without the aid of externally forced air entrainment. The fire whirls that exist before blue-whirl formation are much larger in height than the blue whirl and exist at the laminar-turbulent transition flame regime, evidenced by wrinkled laminar flames at the flame base. In contrast, the blue-whirl regime is characterized by a much smaller length scale (see Fig. 1) and is a laminar flame with no visual or aural indications of turbulence [5,6]. The unexpected transition from a fire whirl to a blue whirl involves laminarization of the flow, which suggests that the interplay between $\dot{Q}$ and $\Gamma$ is different from that in fire whirls.

Lei et al. [4] showed the influence of $\dot{Q}$ and $\Gamma$ on the shape of fire whirls formed in an Emmonstype [2] fire-whirl apparatus where swirl is generated by a spinning mesh around a central flame. Their $\dot{Q}-\Gamma$ map showed the strong influence of $\Gamma$ on the overall shape of fire whirls, especially on the flame shape at the base. This map showed an "extinction limit jump," a sharp increase in the threshold $\dot{Q}$ below which fire whirls were extinguished for all values of $\Gamma$. In recent work, the $\dot{Q}$ - $\Gamma$ map was extended to include the blue-whirl regime [7]. This extended map showed that the combinations of $\dot{Q}$ and $\Gamma$ that led to blue-whirl formation were within the extinction limit defined in Ref. [4]. The formation of blue whirls in this region also requires experimental apparatus to satisfy surface-boundary conditions [8] that were absent in previous work.

In the literature, scaling analyses have often been used to identify parameters and quantities to describe the occurrence of fire whirls [9-12]. The recent extension of this analysis to blue whirls [7] showed that blue whirls exist in a regime distinct from traditional fire whirls. The transition from the fire whirl to the blue whirl was previously hypothesized to be the result of the onset of vortex breakdown [5,13], which is characterized by the presence of a recirculation zone (RZ) [14]. Subsequently, the presence of a RZ was shown to exist in the blue whirl [8], qualitatively confirming these suggestions. The presence of the RZ was observed using streaks of incandescent soot within the conical region of the flame (see Fig. 2). Since soot particles are not present in a stable blue whirl, the $\mathrm{RZ}$ is visible fleetingly during the transition process.

The onset of vortex breakdown is driven by the relative magnitude of local momenta in the axial and azimuthal directions. Different types of vortex breakdown can occur in both nonreacting [15,16] and reacting flows $[17,18]$. The momenta in these directions were compared using a scalar quantity called the swirl number, $\mathcal{S}_{w}$, defined as the ratio of the axial flux of azimuthal momentum to the axial flux of axial momentum [19]:

$$
\mathcal{S}_{w}=\frac{\int_{0}^{R} u_{z} u_{\theta} r^{2} d r}{R \int_{0}^{R} u_{z}^{2} r d r},
$$




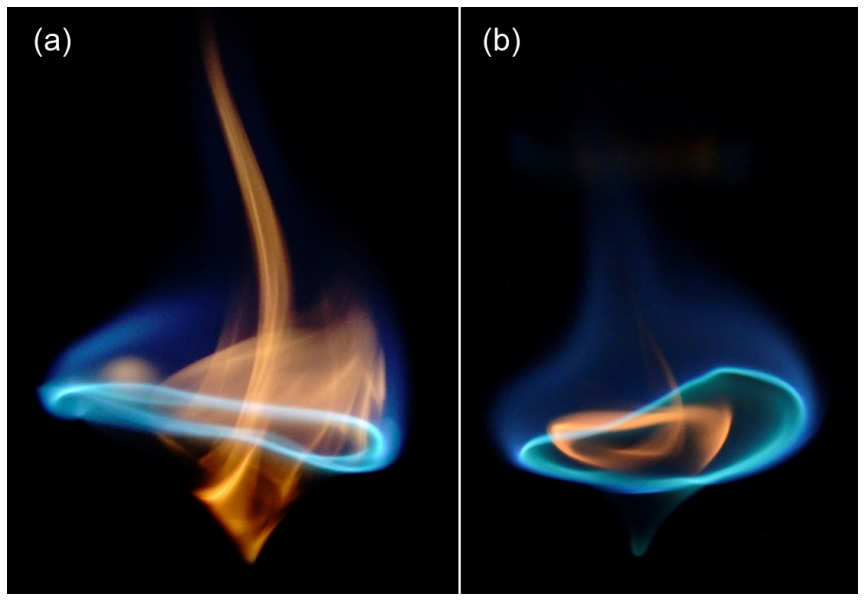

FIG. 2. Images of the blue whirl where the RZ is visualized by streaks of incandescent soot particles. Images (a) and (b) were captured with exposure times of $1 / 60$ and $1 / 100 \mathrm{~s}$, respectively.

where $u_{z}$ and $u_{\theta}$ are the axial and azimuthal velocities, and $R$ is the radius of the vortex core. For an experimental apparatus, however, the definition is simplified and defined based on the geometry of the swirler, with different expressions for axial and radial swirlers [18]. In swirl burners, the RZ develops at a threshold value of $\mathcal{S}_{w} \approx 0.6$ [19].

In fire whirls, the axial and azimuthal momenta are represented by buoyancy and circulation, respectively. The purpose of this work is to test this hypothesis: The flow field of the blue whirl is a state of vortex breakdown that occurs when the local azimuthal momentum becomes higher than the axial momentum, and this blue whirl retains properties of the bubble mode. We approach this from the experimental point of view by reinvestigating the scaling, which helps us quantitatively distinguish between the fire- and blue-whirl regimes. Through this approach, the entrainment conditions required to generate the blue whirl at other length scales are also explored.

Here, we use the raw data for $\dot{Q}$ and $\Gamma$, taken from previous experiments, and define nondimensional quantities to quantify the role played by buoyancy and circulation in whirling flames. These nondimensional quantities are of the same order of magnitude and can be used to differentiate between buoyancy- and circulation-dominated regimes. A nondimensional ratio analogous to $\mathcal{S}_{w}$ is used to establish a threshold value for the onset of vortex breakdown in whirling flames, corresponding to formation of the blue whirl. We find that when the flow field is dominated by buoyancy, the traditional fire whirl develops, and when circulation dominates, there is a transition to the blue whirl.

\section{Background}

In experiments performed to date, formation of a blue whirl has always been preceded by a fire whirl. In addition, a blue whirl can be formed from a variety of different liquid hydrocarbon fuels. Experimental measurements show that it has peak temperatures of about $2000 \mathrm{~K}$ [20-22] and can also be formed on other types of smooth surfaces such as metals [8]. There is intense combustion in the bright blue ring, as evidenced by high concentrations of $\mathrm{OH}, \mathrm{OH}^{*}$, and $\mathrm{CH}^{*}$ radicals in this region $[8,21]$.

Recent experiments [7] allow us to extend previous scaling analyses to include the blue whirl, providing useful context to the material in the current article. In Ref. [7], the experimental setup was a fixed-frame fire-whirl apparatus formed by two semicylindrical quartz segments. The semicylinders form an enclosure within which combustion occurs, and the offset distance between the pieces creates a gap that allows air entrainment into the enclosure. Various combinations of fuel 


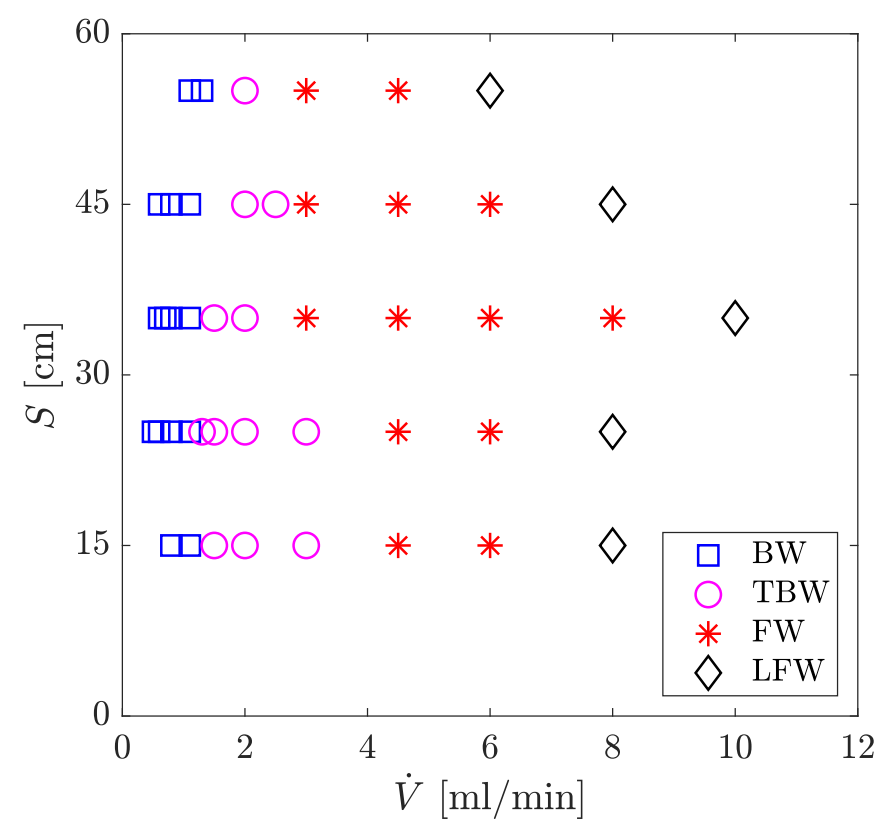

FIG. 3. Influence of $S$ and $\dot{V}$ on the fire-whirl regime, adapted from Ref. [7].

flow rate $(\dot{V})$ and gap size $(S)$ were used to generate fire whirls and blue whirls. The flow velocity into the enclosure was measured at selected axial (vertical) locations using a DANTEC 54T42 Mini CTA anemometer attached to a DANTEC 55P16 hot-wire probe, which was positioned at the center of one gap. These velocity measurements were used to find an approximate circulation for each flame regime according to $\Gamma=\pi U_{\theta} D_{C}$, where $U_{\theta}$ is the tangential velocity measured at the inlet, and $D_{C}$ is the diameter of the enclosure. Assuming complete combustion of the supplied fuel, the heat-release rate was calculated as $\dot{Q}=\dot{V} \Delta h_{c} \rho_{f}$, where $\Delta h_{c}$ is the lower heating value and $\rho_{f}$ is the density of liquid $n$-heptane.

The combination of $S$ and $\dot{V}$ influences the shape of the flame, and three flame regimes were identified: the blue whirl (BW), the stable fire whirl (FW), and the transitional blue whirl (TBW). Images of the different regimes for $S=35 \mathrm{~mm}$ are shown in Fig. 1, and the full data set from Ref. [7] is summarized graphically in Fig. 3. For high values of $\dot{V}$, unsteady FWs were formed with very large diameters, termed as large fire whirls (LFWs). These impeded safe operation of the hot-wire anemometer and were thus avoided in experiments. These raw data were used in a scaling analysis to determine nondimensional quantities for global scaling using the apparatus diameter $\left(D_{C}\right)$ as the characteristic length scale. Although the apparatus diameter or gap size are not intrinsic properties of the FW itself, they have been used in the literature $[9,10]$ for scaling purposes since they influence ambient circulation in FW apparatus with natural air entrainment.

This analysis showed that the BW was close to the extinction limit defined previously [4]. The BW formation limits, defined on the basis of $\dot{Q}$ and $\Gamma$, as well as the relationship between nondimensional heat-release rate $\left(\dot{Q}_{D}^{*}\right)$ and nondimensional circulation $\left(\Gamma_{D}^{*}\right)$, were both sensitive to the gap size between the half-cylinders. The extinction limit for FW regimes was extended by the presence of the BW, and this was attributed to the experimental conditions at the bottom surface over which they formed [8].

Using fixed length scales (such as apparatus or burner diameter) in scaling analyses $[4,23]$ results in quantities that denote general large-scale effects, for instance, the predictions of flame height of FWs for known values of $\Gamma$ and $\dot{Q}$. The use of characteristic length scales derived from flame geometry represent effects of the local flow field. In the present work, the raw data for $\Gamma$ and 


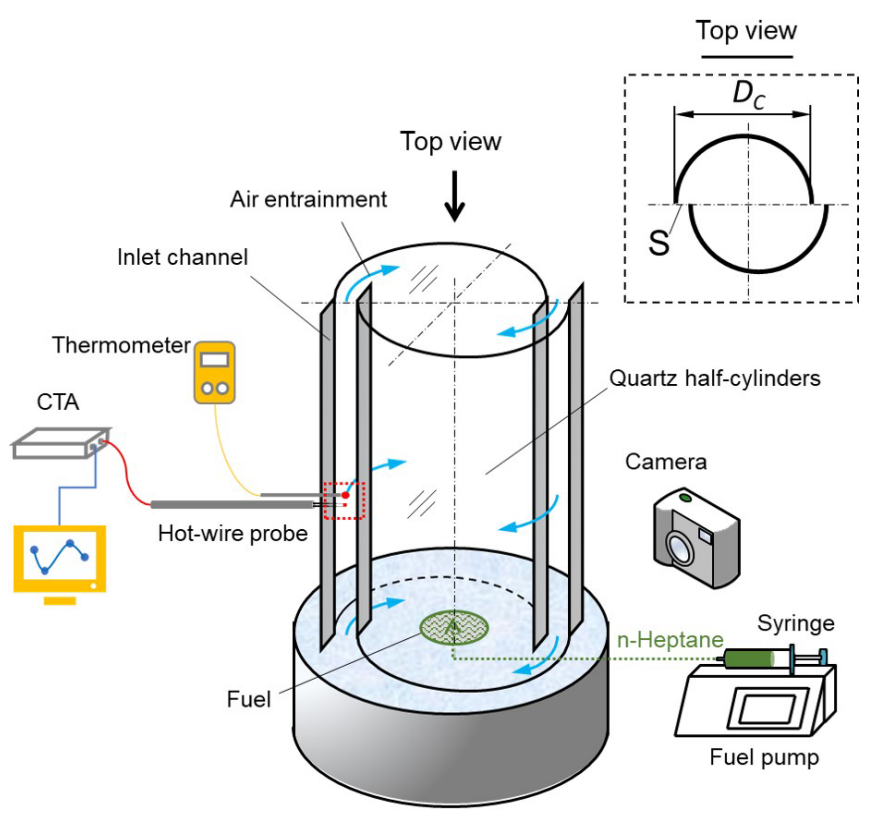

FIG. 4. Schematic of experimental apparatus.

$\dot{Q}$ are combined with additional data on flame geometry. To understand the local effects and the controlling factors in the transition to the BW, the flame height $H$ and the flame width $w_{f}$ are used as characteristic length scales to normalize $\Gamma$ and $\dot{Q}$.

The approach presented in this article helps quantify the effects of the primary competing forces in whirling flames, buoyancy and circulation. By incorporating $H$ and $w_{f}$ into $\Gamma$ and $\dot{Q}$, local buoyancy and circulation in the flame are represented by their respective nondimensional quantities, $\dot{Q}_{f}^{*}$ and $\Gamma_{f}^{*}$. Their ratio then presents a basis for distinguishing buoyancy- and circulation-dominated regimes in whirling flames.

\section{EXPERIMENTAL METHODS}

The experimental apparatus (Fig. 4), similar to that used in previous work [5,7,8,20], consisted of two quartz half-cylinders (310 $\mathrm{mm}$ in diameter, $600 \mathrm{~mm}$ in height) suspended on an aluminum frame. The two quartz pieces were positioned over a water pan and offset from each other, forming gaps for natural entrainment of air. Liquid fuel, here $n$-heptane, was supplied to the water surface using a syringe pump at a constant volumetric flow rate. Upon ignition of the fuel, the buoyant flow due to heat release within the enclosure draws air into the enclosure through the two gaps. For all experiments, a short overlap region was constructed from thin sheets of aluminum to form a channel at each inlet (see Fig. 4). The effect of this inlet channel is discussed in Sec. III. All data were obtained with the presence of these overlapping inlet channels.

A typical experiment involved the injection of $10 \mathrm{ml}$ of fuel onto the water surface, followed by ignition using a propane torch. Initially, a pool fire was formed and lasted for a few seconds before evolving to a FW. Upon transition to a fire whirl, fuel was supplied at a constant rate using the syringe pump. After about $60 \mathrm{~s}$, the rate of fuel supply (from the syringe pump) and consumption (by the FW) were nearly equal, and a stable FW or BW formed, depending on the gap size and the fuel supply rate. For a given gap size, $S$, the regime of the FW depended on the fuel supply rate $\dot{V}$, which was varied in the range of $[0.5,10.0] \mathrm{ml} / \mathrm{min}$. $S$ was varied in the range $[15.0,55.0] \mathrm{mm}$. 
Due to the natural-entrainment configuration, the only regimes that formed resulted from a balance between these parameters.

Measurements were made of the flame geometry for each regime. Videos $(1280 \times 720$ pixel resolution) of each experiment were recorded at $60 \mathrm{fps}$ for $60 \mathrm{~s}$ using a Sony RX10II, with an aperture of $f / 5.6$. The flame width $\left(w_{f}\right)$ and the flame height $(H)$ were obtained by averaging the flame contour on multiple frames. From each 60-s video, three separate periods of 0-12 s, 24-36 s, and 48-60 s were chosen, and 720 individual frames were extracted from each period. The resulting 2160 RGB images for each experimental condition were converted into grayscale images, which were then converted to a binary format in IMAGEJ [24] using Otsu's method [25] to determine a binarization threshold. This method was reliable since exposure in the videos was set such that there was a dark background in contrast to the bright flame, resulting in maximum intensity separation at the edge of the flame sheet. The mean binary value for each pixel was obtained by stacking all 720 binary images for each time period. Dividing the mean binary value by 256 (the range of intensity values in grayscale), the probability of the flame appearance for each pixel was determined, giving the probability contours of the flame. The mean flame contour was defined by a probability of 0.5 based on previous literature [26].

Based on this mean contour, the flame width measured at the widest cross section, was found $\sim 10 \mathrm{~mm}$ above the water surface for FWs, and the width of the bright blue ring (also called the vortex rim in Ref. [20]) was used as the flame width for both the BW and the TBW. The flame height was measured at the highest position of the continuous flame region above the water surface. For each time period, one set of $w_{f}$ and $H$ was obtained, and an overall average was obtained by calculating a mean from the values for the three time periods recorded and binarized.

\section{DEPENDENCE OF FLAME GEOMETRY ON $\dot{Q}$ AND $\Gamma$}

Figure 5 shows the variation of the flame geometry parameters $w_{f}$ and $H$ with both $\Gamma$ and $\dot{Q}$. The narrow error bars for the FW and BW regimes reflect the relative stability of their flame geometry during the 60-s period analyzed [Figs. 5(a) and 5(b)]. TBWs, on the other hand, show significant changes in flame geometry parameters due to their repeated transitions between the FW and BW regimes. The FW shows the highest values of $w_{f}$, which is not a strong function of $\Gamma$ or $\dot{Q}$. The dashed line in Fig. 5(c) shows a slight decrease from the BW to the TBW, followed by an increase for FWs.

The value of $H$, however, varies with both $\Gamma$ and $\dot{Q}$. The increase in $H$ with $\Gamma$ is shown in Fig. 5(b). The curve is approximated by the fit in Eq. (1), which has an $R^{2}$ value of 0.55 . The fit in Eq. (1) does not include data points for $S=15 \mathrm{~mm}$ in Fig. 5(c). The data points for $S=15 \mathrm{~mm}$ (solid square markers) follow a trend different from the other conditions. This bifurcation behavior was discussed in previous work [7]. The relationship between $H$ and $\dot{Q}$, shown in Fig. 5(d), is given by Eq. (2), which has an $R^{2}$ (coefficient of determination) value of 0.96 . Equations (1) and (2) are given as follows:

$$
\begin{gathered}
H=52.03 \Gamma^{3.89}, \\
H=2.91 \dot{Q}^{1.19} .
\end{gathered}
$$

The effect of the inlet channels (see Fig. 4) on the fluctuation in $H$ for BWs is shown in Fig. 6. The BW is particularly sensitive to ambient perturbations, which cause $H$ to fluctuate significantly when the inlet channel is not present. This results in the large variations in $H$, seen in Fig. 6(a), particularly near the extinction and transition limits. When the inlet channel was present, the variation in $H$ for BWs decreased even in conditions close to the extinction and transition limits [Fig. 6(b)]. Furthermore, the BW formation limits were also extended when the inlet channels were present. The differences between Figs. 6(a) and 6(b) reflect the role of the inlet channels in stabilizing the BW. The experimental data obtained with the inlet channel are used below in the scaling analysis since they correspond to more stable experimental conditions. 
(a)

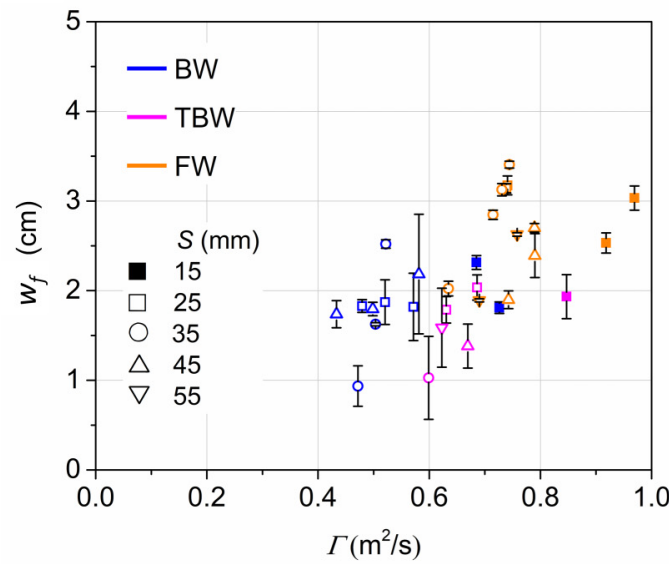

(c)

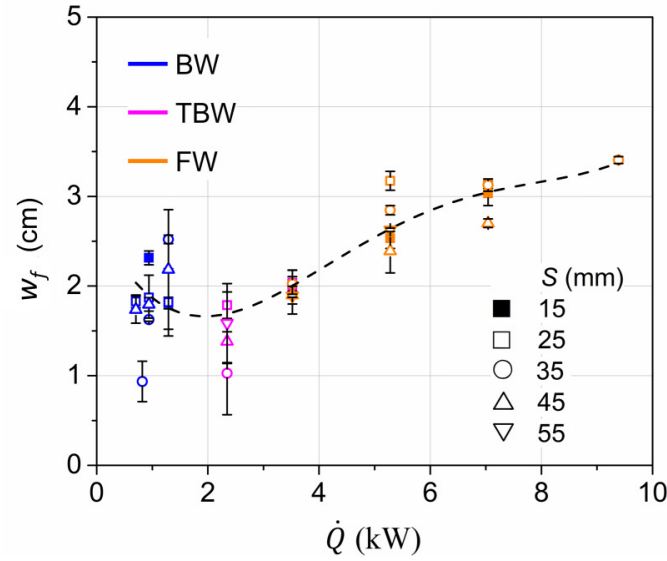

(b)

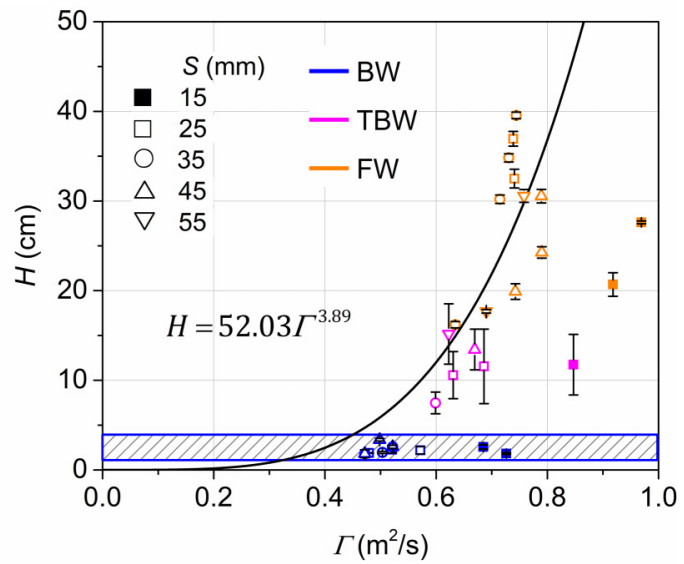

(d)

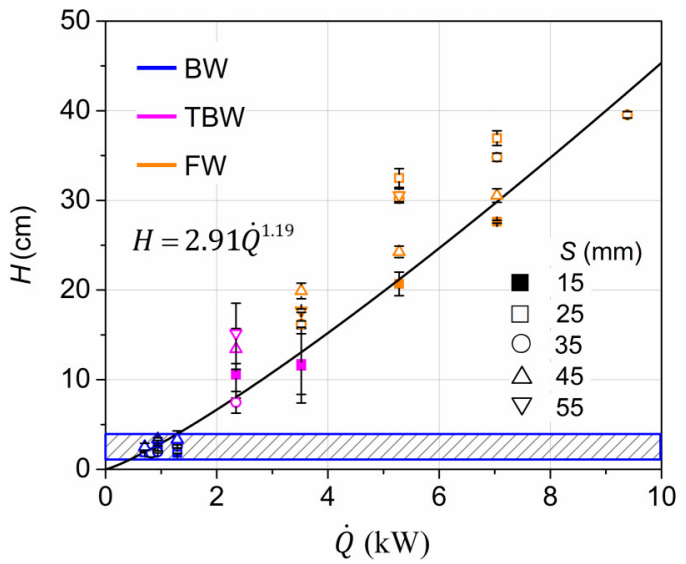

FIG. 5. Variation of $w_{f}$ (a), (b) and $H$ (c), (d) with $\Gamma$ and $\dot{Q}$. The hashed section in panels (b) and (d) indicates the limited variation in $H$ for the BW. In panel (b), flame regimes at $S=15 \mathrm{~mm}$ are not considered for the curve fit shown and follow a different trend. This difference at low $S^{*}$ is discussed in Ref. [7].

\section{DIMENSIONAL ANALYSIS}

Based on a review of previous scaling methods used for FWs [11], the parameters governing circulation $(\Gamma)$ of a FW in a fixed-frame-type apparatus are

$$
\Gamma=f_{1}\left(\dot{Q}, S, w_{f}, H, D_{C}, T_{0}, \rho_{0}, \Delta T, \Delta \rho, C_{p, 0}, g\right)
$$

where $\Gamma, \dot{Q}, S, w_{f}, H$, and $D_{C}$ are as defined previously. The quantities $T_{0}, \rho_{0}, \Delta T, \Delta \rho, C_{p, 0}$, and $g$ are ambient temperature, ambient density, excess temperature, change in density, specific heat of air at $T_{0}$, and gravitational acceleration, respectively. Equation (3) assumes that (i) circulation is independent of axial location of the $\mathrm{FW}$, (ii) viscosity is negligible relative to inertial and buoyant forces, and (iii) combustion is infinitely fast and steady.

As shown in Fig. 1, the geometry of the flame may be used to distinguish among the different flame regimes. Specifically, $H$ strongly depends on $\Gamma$ and $\dot{Q}$ (Fig. 5). Using $H$ and $w_{f}$ as characteristic length scales, $D_{C}, T_{0}, \rho_{0}$, and $g$ were chosen as the basic physical parameters to apply the 
(a)

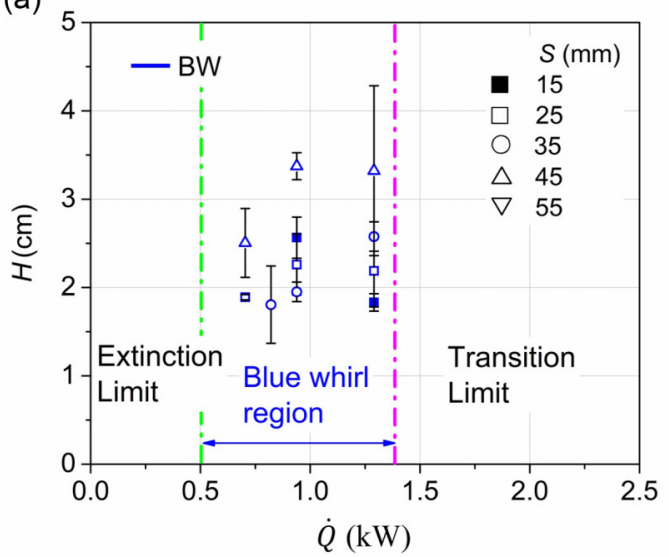

(b)

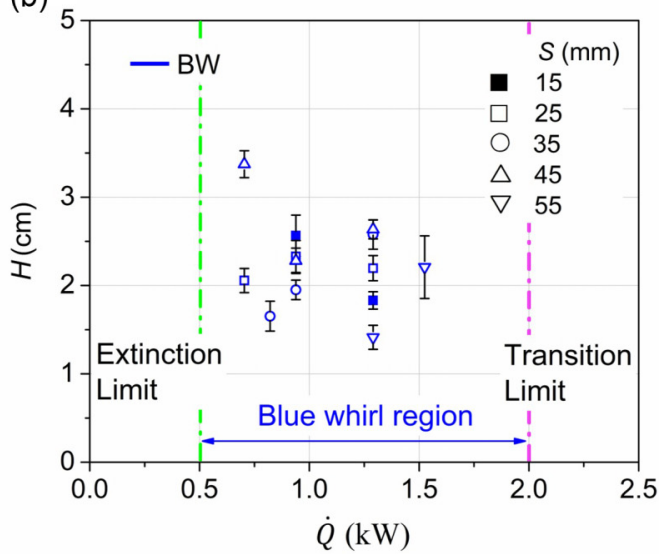

FIG. 6. Variation of $H$ with $\dot{Q}$, shown for cases (a) without and (b) with the inlet channels for the BW. The vertical dashed lines, green (left) and magenta (right), indicate limits of extinction and transition, respectively.

Buckingham $\Pi$ theorem [27] on Eq. (3), yielding eight nondimensional $\Pi$ terms shown below:

$$
\left(\Gamma_{f}^{*}, H^{*}, \frac{w_{f}}{D_{C}}\right)=f_{2}\left(\dot{Q}_{f}^{*}, S^{*}, \frac{\Delta T}{T_{0}}, \frac{\Delta \rho}{\rho_{0}}, \frac{g w_{f}}{C_{p, 0} \Delta T}\right) .
$$

The dimensionless circulation is defined as $\Gamma_{f}^{*}=\left[\Gamma /\left(D_{C} \sqrt{g H}\right)\right]=\left(\pi U_{\theta} / \sqrt{g H}\right)$, which is analogous to the Froude number, defined as $\mathrm{Fr}=\left(U^{2} / g d\right)$. The quantity $\dot{Q}_{f}^{*}=\dot{Q} /\left(C_{p, 0} \Delta T \rho_{0} w_{f}^{2} \sqrt{g H}\right)$ is the dimensionless heat-release rate, representing the ratio of the actual heat-release to a reference combustion enthalpy $\left[\dot{V} /\left(w_{f}^{2} \sqrt{g H}\right)\right]$, where the subscript " $f$ " denotes normalization by a flame dimension. In the quantity $\dot{Q}_{f}^{*}$, the denominator $\left(C_{p, 0} \Delta T \rho_{0} w_{f}^{2} \sqrt{g H}\right)$ denotes the volumetric distribution of the heat-release rate for a nonswirling diffusion flame of width $w_{f}$ and height $H$. Thus, $\dot{Q}_{f}^{*}$ reflects an enhancement in combustion intensity due to the presence of swirl and also represents the buoyancy due to heat release. This is discussed further in Sec. V.

The ratio $S^{*}=S / D_{C}$ is the dimensionless gap size, a geometric feature of the setup. The quantity $\left(w_{f} / D_{C}\right)$ is nearly constant for a given regime (see Fig. 5). The quantities $\left(\Delta T / T_{0}\right)$ and $\left(\Delta \rho / \rho_{0}\right)$ can be assumed to be nearly constant for most fires [28], and $\left(g w_{f} / C_{p, 0} \Delta T\right)$ indicates the ratio of potential energy to thermal energy, which is small enough to be neglected here. Hence, Eq. (4) reduces to

$$
\begin{gathered}
\left(\Gamma_{f}^{*}, H^{*}\right)=f_{3}\left(\dot{Q}_{f}^{*}, S^{*}\right), \\
H^{*}=f_{4}\left(\Gamma_{f}^{*}, \dot{Q}_{f}^{*}, S^{*}\right),
\end{gathered}
$$

where $H^{*}=H / w_{f}$.

According to Eq. (6), $H^{*}$ depends on $\Gamma_{f}^{*}, \dot{Q}_{f}^{*}$, and $S^{*}$. Figure 7(a) shows that $\dot{Q}_{f}^{*}$ has minimal influence on $H^{*}$, with large differences between the values for the BW and the FW. So, $H^{*}$ changes only when the flame regime changes, and for each flame regime, the influence of $\dot{Q}_{f}^{*}$ is limited. This is particularly true for the BW regime, which has a very narrow band of $H^{*}$. For a given flame regime, $H^{*}$ does not show any trend with $S^{*}$.

The quantity $H^{*}$, however, does depend on $\Gamma_{f}^{*}$, as shown in Fig. 7(b). Figure 7 shows that there is a difference of an order of magnitude in $H^{*}$ between the $\mathrm{BW}$ and $\mathrm{FW}$ regimes. The solid line in Fig. 7(b) shows the power-law relationship between $H^{*}$ and $\Gamma_{f}^{*}$, approximated by Eq. (7) with an 

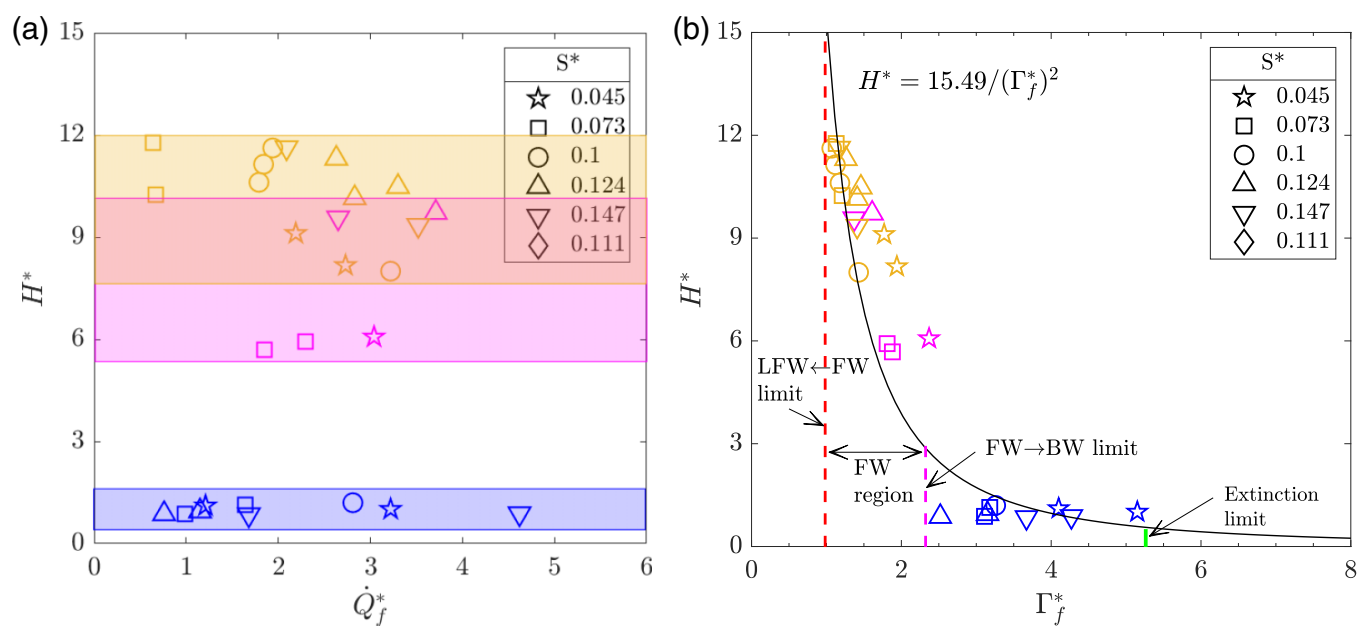

FIG. 7. (a) Variation of $H^{*}$ with $\dot{Q}_{f}^{*}$. The range of $H^{*}$ for the BW and the FW do not overlap, showing that it varies only when the regime changes. The colored stripes denote the range of $H^{*}$ for each regime. $S^{*}$ does not influence $H^{*}$, particularly in the BW regime. (b) Variation of $\Gamma_{f}^{*}$ with $H^{*}$, with the different transition limits shown as dotted lines.

$R^{2}$ of 0.9:

$$
H^{*}=15.49 /\left(\Gamma_{f}^{* 2}\right)
$$

When compared to FWs, BWs form in a region of relatively high $\Gamma_{f}^{*}$ and low $H^{*}$ [Fig. 7(b)]. $\mathrm{BW}$ formation occurs for $\Gamma_{f}^{*} \in[2.4,5.2]$, while FWs generally form in a much narrower region where $\Gamma_{f}^{*} \in[1.0,1.4]$. The FWs for $S^{*}=0.045$ deviate slightly from this trend and occur closer to $\Gamma_{f}^{*} \approx 2$. Below $\Gamma_{f}^{*}=2.4$, which is the BW transition limit in Fig. 7(b), $H^{*}$ begins to increase rapidly. Generally, these results show that the dimensionless circulation $\Gamma_{f}^{*}$ has a significant impact on $H^{*}$ and appears in this analysis as an important parameter to understand the transition between the FW and BW flame regimes.

The LFW limit is near $\Gamma_{f}^{*}=1$. The LFW was unsteady and its diameter was comparable to that of the enclosure, which did not permit any measurements. Consequently, below we use data for LFWs from the literature to calculate the required nondimensional quantities. In the following section, we focus on the relationship between $\Gamma_{f}^{*}$ and $\dot{Q}_{f}^{*}$, both of which are $\mathcal{O}(1)$.

\section{DISCUSSION}

The three nondimensional quantities $\Gamma_{f}^{*}, \dot{Q}_{f}^{*}$, and $H^{*}$ have different relationships for the FW and BW regimes and may be used to provide insight on the local effects governing the transition from FWs to BWs. Going from the BW to the FW, while $w_{f}$ is relatively constant with $\dot{V}, H$ increases continuously. Thus, $H$ and $H^{*}$ distinguish these regimes. The near-linear increase in $H$ with $\dot{V}$ is similar in behavior to laminar jet diffusion flames [29]. Within the BW regime, however, $H$ varies little with $\dot{V}$. Figures 5(c) and 5(d) show that the BW forms in a region of low $\dot{Q}$. Since entrainment velocity is directly proportional to $\dot{Q}$ (see Fig. 5 in Ref. [7]), this leads to low $\Gamma$ for BWs. $\dot{Q}_{f}^{*}$, however, is comparable for FWs and BWs [Fig. 7(a)].

Figure 7(b) indicates that the BW forms in a region where $\Gamma_{f}^{*}$ is relatively high. As $\Gamma_{f}^{*}$ decreases, there is a steep increase in $H^{*}$, leading to the FW. With further decrease in $\Gamma_{f}^{*}$, the effect of circulation on the buoyant plume reduces. LFWs are formed when $\Gamma_{f}^{*} \in(0,1)$ and flame height varies roughly linearly with circulation $[4,10]$. Eventually, when $\Gamma_{f}^{*}=0$, a free-convection pool-fire is formed [30,31]. Here, $H^{*}$ depends on other factors such as $\dot{Q}$ and the pool diameter [32,33]. 


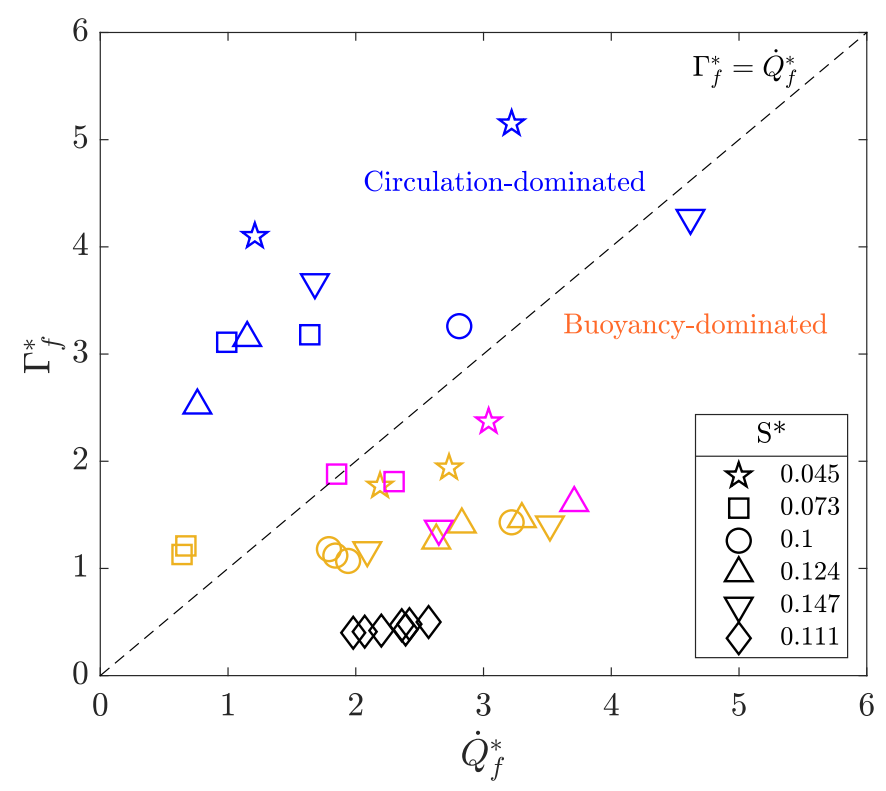

FIG. 8. $\Gamma_{f}^{*}$ as a function of $\dot{Q}_{f}^{*}$ for the different regimes in this study. Also included are markers for LFWs, calculated from data in Table 1 in Lei et al. [34], whose experiments used a propane burner within a square cross-section setup, with natural air entrainment at $S^{*}=0.111$ (calculated based on the definition in this study) and $\dot{Q} \in[25,300] \mathrm{kW}$. The blue markers correspond to BWs, magenta to TBWs, yellow to FWs, and black to LFWs.

Plotting $\dot{Q}_{f}^{*}$ vs $\Gamma_{f}^{*}$ allows for the different regimes to be more clearly distinguished (see Fig. 8). Since measurements of the LFW (at $\Gamma_{f}^{*}<1$ ) are not available from this work, data of LFWs from the literature are used for comparison. Fire whirls similar to the LFW were investigated by Lei et al. [34] using a propane burner in a square fixed-frame setup. The LFWs in their experiments form in a narrow range of $\Gamma_{f}^{*}$, lower than the FWs in this study, and are also included for comparison in Figs. 8 and 9.

The quantities $\dot{Q}_{f}^{*}$ and $\Gamma_{f}^{*}$ are of the same order and represent buoyancy and circulation, respectively. The dashed line in Fig. 8 represents $\Gamma_{f}^{*}=\dot{Q}_{f}^{*}$, where the influence of circulation and buoyancy is roughly balanced. For the flame regimes above this line, circulation dominates (i.e., $\Gamma_{f}^{*}>\dot{Q}_{f}^{*}$ ), and for those below, buoyancy dominates (i.e., $\Gamma_{f}^{*}<\dot{Q}_{f}^{*}$ ). In general, the BW lies above the $\Gamma_{f}^{*}=\dot{Q}_{f}^{*}$ line, and FWs lie in the region below. Data points for LFWs are well within the buoyancy-dominated region. Figure 9(a) and Table I show that for naturally entrained FWs, with increasing scale, buoyancy tends to dominate circulation (see markers for LFW). This is a consequence of larger $\dot{Q}$, which for liquid-fueled FWs is partially controlled by the fuel pool diameter.

Of the five data points available for the TBW regime, three lie between the FW and BW regimes. This transition regime is expected to occur in the neighborhood of $\Gamma_{f}^{*}=\dot{Q}_{f}^{*}$, although Fig. 8 shows two instances deviating more towards the FW side (these deviations are observed for one instance of the BW at $S^{*}=0.147$ and two FWs at $S^{*}=0.073$ ). This spread in TBW data is expected from the large fluctuations in $H$ in this regime [see Figs. 5(c) and 5(d)]. Additionally, only five data points exist for the TBW regime in our work, and the trend may become more apparent when there are more data available.

The quantity $\left(\Gamma_{f}^{*}\right)^{2}$ is the Froude number, Fr. While Fr represents the competition between buoyant and external momentum, it does not fully represent the effect of circulation on $H$. Chuah 

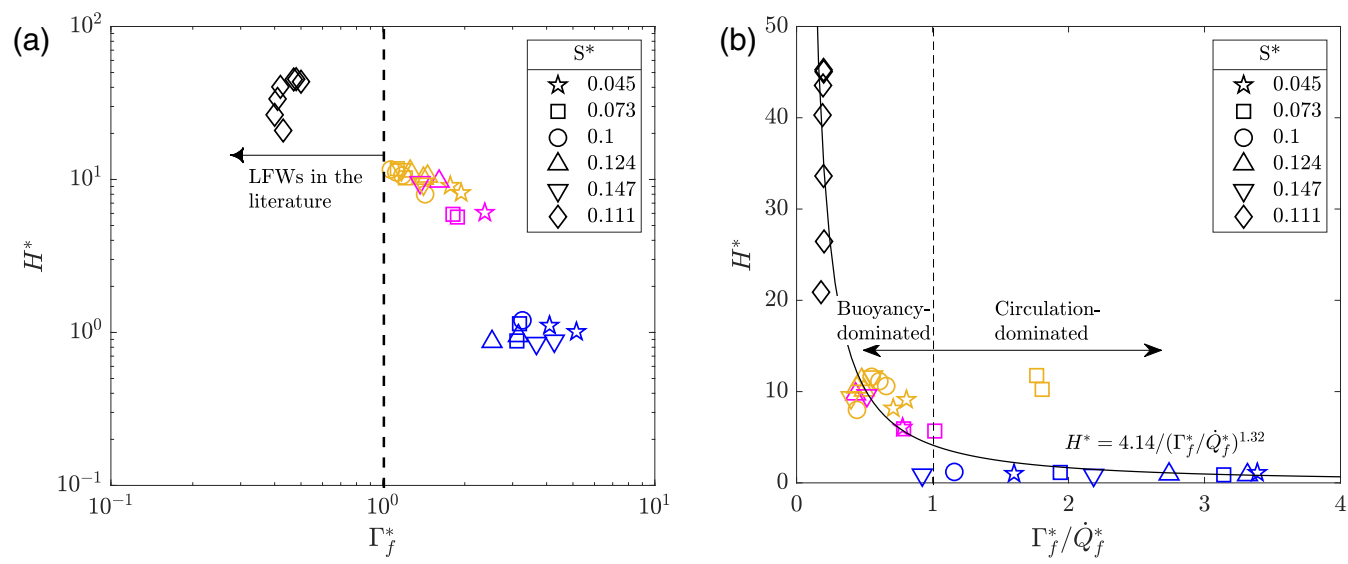

FIG. 9. Comparison of experimental data in this work with those for LFWs in the literature [34]. (a) The LFW regime occurs when $\Gamma_{f}^{*} \in(0,1)$. The values of $H^{*}$ for LFWs in the literature are many times those of the FWs in this study and an order of magnitude higher than the BWs. (b) The FW-to-BW transition occurs around $\Gamma_{f}^{*} / \dot{Q}_{f}^{*} \approx 1$ in the circulation-dominated regime.

et al. [28] pointed out that the value of Ro (Rossby number) in relation to Fr was important for quantifying the effect of circulation on the burning rate in whirling flames. This is especially applicable to configurations with natural air entrainment where $\Gamma$ and $\dot{Q}$ are not independent.

While $\Gamma_{f}^{*}$ varies significantly for the BW and FW regimes, it does not help in comparing the local relationship between axial and buoyant momenta. Thus for whirling flames, it is more useful to represent the competition between circulation (tangential component) and buoyancy (axial component $)$ in the form of the ratio $\mathcal{R}^{*}=\left(\Gamma_{f}^{*} / \dot{Q}_{f}^{*}\right)$. The value of this ratio for the different regimes is shown in Table I. Figure 9(b) shows the relationship between $H^{*}$ with $\left(\mathcal{R}^{*}\right)$ for all the regimes. The relationship is approximated by a least-squares fit as $H^{*}=4.14 /\left(\mathcal{R}^{*}\right)^{1.32}$. This graph shows that the transition to the BW occurs in the neighborhood of $\mathcal{R}^{*}=1$, when circulation begins to dominate buoyancy. In other reacting or nonreacting swirl flows, this condition leads to the formation of a recirculation zone (RZ) and to vortex breakdown [17,19]. In swirl burners, the threshold value for $\mathrm{RZ}$ formation is $\mathcal{S}_{w}=0.6$ [18].

Earlier work showed the presence of a RZ within the BW and suggested that the shape of the BW regime may be governed by the bubble mode of vortex breakdown [20,35]. While the RZ is visible during the transition process, it is not visible in a stable BW due to the absence of soot tracers [8]. Assuming that the development of a RZ is necessary for BW formation [13], $\mathcal{R}^{*}$ is analogous to $\mathcal{S}_{w}$ in predicting the onset of vortex breakdown in whirling flames and may therefore represent an appropriate nondimensional scale to distinguish whirling flames. Table I shows that the threshold value of $\mathcal{R}^{*}$ leading to vortex breakdown and BW formation is $\approx 1$.

The large difference in the values of $\mathcal{R}^{*}$ for FWs and BWs provides hints as to why the BW regime was not observed in previous FW experiments. In addition to the smooth bottom boundary without obstructions to the incoming radial flow [8], a circulation-dominated regime $\left(\Gamma_{f}^{*}>\dot{Q}_{f}^{*}\right)$ is required for transition to the BW. FWs subject to strong vorticity have been studied previously $[28,36]$ and it was found that elongation in FW flame length, when compared to a pool fire, cannot be fully attributed to increasing burning rates. This suggests that that the vortex structure, within which fuel fractions are high, plays a significant role in the increased flame height.

In the case of transition from FWs to BWs, increasing $\Gamma_{f}^{*}$ results in suppression of $H^{*}$, eventually leading to BW formation [Figs. 7(b) and 9]. The BW does not show much variation in $H^{*}$ [see Fig. 7(a)], which may be attributed to the existence of a RZ upon vortex breakdown. The RZ potentially aids in better mixing, leading to a smaller volume required for the reaction to occur and 
TABLE I. Values of $\mathcal{R}^{*}=\left(\Gamma_{f}^{*} / \dot{Q}_{f}^{*}\right)$ for the BW, TBW, FW, and LFW regimes.

\begin{tabular}{|c|c|c|c|c|}
\hline \multirow[b]{2}{*}{$S^{*}$} & \multicolumn{4}{|c|}{$\mathcal{R}^{*}$} \\
\hline & BW & TBW & FW & LFW \\
\hline $\begin{array}{l}0.045 \\
(S=15 \mathrm{~mm})\end{array}$ & $\begin{array}{l}3.39 \\
1.6\end{array}$ & 0.78 & $\begin{array}{l}0.71 \\
0.81\end{array}$ & \\
\hline $\begin{array}{l}0.073 \\
(S=25 \mathrm{~mm})\end{array}$ & $\begin{array}{l}3.15 \\
1.94\end{array}$ & $\begin{array}{l}0.78 \\
1.02\end{array}$ & $\begin{array}{l}1.82 \\
1.78\end{array}$ & \\
\hline $\begin{array}{l}0.099 \\
(S=35 \mathrm{~mm})\end{array}$ & 1.16 & & $\begin{array}{l}0.44 \\
0.66 \\
0.61 \\
0.55\end{array}$ & \\
\hline $\begin{array}{l}0.124 \\
(S=45 \mathrm{~mm})\end{array}$ & $\begin{array}{l}3.33 \\
2.73\end{array}$ & 0.43 & $\begin{array}{l}0.44 \\
0.5 \\
0.48\end{array}$ & \\
\hline $\begin{array}{l}0.147 \\
(S=55 \mathrm{~mm})\end{array}$ & $\begin{array}{l}0.92 \\
2.18\end{array}$ & 0.52 & $\begin{array}{l}0.4 \\
0.56\end{array}$ & \\
\hline $\begin{array}{l}0.111 \\
(S=300 \mathrm{~mm}) \\
\text { From [34] }\end{array}$ & & & & $\begin{array}{l}0.18 \\
0.2 \\
0.2 \\
0.19 \\
0.2 \\
0.2 \\
0.19\end{array}$ \\
\hline
\end{tabular}

causing suppression of $H^{*}$. This is similar to the reduction in flame height of laminar jet diffusion flames upon transition to turbulence [29].

Coenen et al. [13] noted that for a given fuel pool diameter, BW formation required evaporation of fuel from a fraction of the total pool area such that $\dot{Q}$ and $H$ decrease simultaneously. The contraction in evaporation area is a consequence of intensification in vorticity as the width of the FW vortex core reduces. This behavior agrees with the data from the present work, where decreasing $H^{*}$ and $\dot{Q}_{f}^{*}$ is enabled by increasing $\Gamma_{f}^{*}$. Since circulation is conserved radially [2], a reduced vortex core diameter is formed due to tangential momentum increasing relative to axial momentum locally, resulting in the conditions required for a vortex breakdown bubble to form and resulting in the BW. The limits presented in this work are determined from experiments on regimes formed by natural air entrainment. Controlling the transition process or further expanding the envelope of BW formation conditions, if possible by means of external forced entrainment, will require independent control of $\dot{Q}, \Gamma$, and the fuel pool area to ensure $\Gamma_{f}^{*}>\dot{Q}_{f}^{*}$.

For all of the flame regimes, the influence of $\Gamma$ and $\dot{Q}$ is weak on $w_{f}$ but strong on $H$. The flame dimensions are determined by the axial flux of fuel vapor, which is determined by $\dot{V}$, and thus the diameter of the fuel pool over the water surface. For a given flame regime, the fuel mass flux upon evaporation is nearly constant, and the increase in specific volume upon combustion of the fuel vapors shows a near-linear growth in $H$ rather than $w_{f}$. This is similar to the behavior of jet diffusion flames, for which the flame height in the laminar regime depends on the volumetric flow rate of fuel from the fuel port, before a transition to turbulence reduces the flame height (see Fig. 5.10 in Ref. [29]).

Whether the blue whirl can be formed at much larger values of $D_{C}$, and consequently $w_{f}$ and $H$ (or $\Gamma$ and $\dot{Q}$ ), is an open question. Transition to turbulence at high values of $\dot{Q}$ (and thus high 
Grashof number) are expected, and natural (or forced) entrainment at high tangential velocity may prevent BW formation. The scaling approach in this work is applicable in the range of the data presented and may be extended to larger length scales (and be of a predictive nature) only if the relationship between circulation and buoyancy remain the same with increasing values of $\Gamma$ and $\dot{Q}$ at larger values of $D_{C}$. In any case, the transition is still expected to be expressed in a form similar to $\Gamma_{f}^{*}=\varphi\left(\dot{Q}_{f}^{*}\right)$, possibly with different characteristic length or time scales, since they represent the two fundamental properties that determine the regime of a whirling flame.

\section{SUMMARY AND CONCLUSION}

The transition from fire whirls to blue whirls was studied by a scaling approach. Using experimental data, an investigation of the scaling parameters now allows us to understand the relative influences of circulation and buoyancy in determining the different fire-whirl regimes. In addition to data from previous work, additional measurements of the flame geometry (height, $H$, and width, $w_{f}$ ) were obtained from videos.

The flame width did not vary significantly with circulation $(\Gamma)$ or heat-release rate $(\dot{Q})$ for the different flame regimes. The relationship between $H$ and $\Gamma$ was exponential and between $H$ and $\dot{Q}$ was nearly linear. Using $H$ and $w_{f}$ as the characteristic length scales, two primary nondimensional quantities were defined: dimensionless heat-release rate, $\dot{Q}_{f}^{*}$, and dimensionless circulation, $\Gamma_{f}^{*}$. These quantities were of the same order of magnitude and represent the effects of buoyancy and circulation in whirling flames.

The ratio of these quantities, $\mathcal{R}^{*}=\left(\Gamma_{f}^{*} / \dot{Q}_{f}^{*}\right)$, represents the relative influences of circulation and buoyancy on the flame. This is analogous to the swirl number for swirling jets, where the relative magnitudes of axial Reynolds number and azimuthal swirl determine the flame regime. The influence of this ratio on the transition may be summarized as

$$
\mathcal{R}^{*}=\left\{\begin{array}{lll}
<1, & \text { buoyancy dominated, } & \mathrm{FW} \\
\approx 1, & \text { transitional, } & \mathrm{TBW} \\
>1, & \text { circulation dominated, } & \mathrm{BW}
\end{array}\right.
$$

A value of $\mathcal{R}^{*}<1$ represents a flow field dominated by buoyancy, and most fire whirls belong to this regime, with values in the range [0.4, 0.8]. Two instances of fire whirls (referred to as "conical fire whirls" in Ref. [4]) showed an average value of 1.8. The value of this ratio was calculated to be $\sim 0.2$ for large fire whirls in the literature, placing them well within the buoyancy-dominated regime.

For the blue whirls in this study, $\mathcal{R}^{*} \in[0.9,3.4]$, and generally $\mathcal{R}^{*}>1$. This indicates a flow regime where circulation dominates over the buoyancy locally. The transitional blue whirl is defined as a regime that continuously switches between the blue whirl and the fire whirl and may theoretically be expected to occur when the ratio is in the neighborhood of 1 , where the effects of buoyancy and circulation are roughly equal. The number of data points for transitional blue whirls was limited and calculated to be in the range of $[0.43,1]$. This range extends more towards the buoyancy-dominated side and is attributed to the large fluctuations in $H$, caused by repeated alternation between the fire-whirl and blue-whirl regimes.

The transition from the fire whirl to the blue whirl occurs at a threshold value of $\mathcal{R}^{*}=1$, roughly where circulation begins to dominate buoyancy. This favors the formation of a recirculation zone, leading to the onset of vortex breakdown. This is one reason why the blue whirl may not have been discovered in previous apparatuses. Previous fire-whirl apparatuses generated only buoyancy-dominated regimes by design and did not provide the strong radial inflow at the bottom boundary surface [8]. The right combination of low $\dot{Q}$ and natural entrainment at the right length scale provided the optimal conditions for its discovery in the experiments performed by Xiao et al. [5]. With the results presented here, future experiments of the blue whirl can be designed with forced entrainment apparatus to control $\dot{Q}$ and $\Gamma$ independently at different length scales to directly hone in on the BW regime. 
This work provides a quantitative basis to explain the factors controlling the transition from the fire whirl to the blue whirl and lays the foundation for future experimental and numerical efforts on fire whirls and blue whirls. Open questions still remain regarding the flow structure within the recirculation zone and the mixing conditions that stabilize the flame at the bright blue ring. Future measurements using nonintrusive laser diagnostics of the flow-field in the vicinity of the flame and the distribution of radical species will be helpful in further understanding these better.

\section{ACKNOWLEDGMENTS}

This work was funded by the National Science Foundation (NSF, Grants No. CBET-1507623 and CBET-1554026), the Texas A\&M University through TEES Eminent Research Professorship, China Scholarship Council (CSC, Grant No. 201606210356), and the Bureau of Safety and Environmental Enforcement (BSEE, Contract No. E17PC00016). Opinions, findings, conclusions, and recommendations expressed here are those of the authors and do not necessarily reflect the views of the NSF, the CSC or the BSEE.

The authors declare that they have no known competing financial interests or personal relationships that could have appeared to influence the work presented here.

[1] G. M. Byram and R. E. Martin, Fire whirlwinds in the laboratory, Fire Control Notes 23, 13 (1962).

[2] H. W. Emmons and S.-J. Ying, The fire whirl, Symp. (Int.) Combus. 11, 475 (1967).

[3] G. M. Byram and R. E. Martin, The modeling of fire whirlwinds, For. Sci. 16, 386 (1970).

[4] J. Lei, N. Liu, Y. Jiao, and S. Zhang, Experimental investigation on flame patterns of buoyant diffusion flame in a large range of imposed circulations, Proc. Combust. Institute 36, 3149 (2017).

[5] H. Xiao, M. J. Gollner, and E. S. Oran, From fire whirls to blue whirls and combustion with reduced pollution, Proc. Natl. Acad. Sci. USA 113, 9457 (2016).

[6] S. B. Hariharan, The structure of the blue whirl: A soot-free reacting vortex phenomenon, M.S. Thesis, University of Maryland, College Park, 2017.

[7] Y. Hu, S. B. Hariharan, H. Qi, M. J. Gollner, and E. S. Oran, Conditions for formation of the blue whirl, Combust. Flame 205, 147 (2019).

[8] S. B. Hariharan, P. M. Anderson, H. Xiao, M. J. Gollner, and E. S. Oran, The blue whirl: Boundary layer effects, temperature and $\mathrm{OH}^{*}$ measurements, Combust. Flame 203, 352 (2019).

[9] K. Kuwana, K. Sekimoto, K. Saito, and F. A. Williams, Scaling fire whirls, Fire Saf. J. 43, 252 (2008).

[10] K. Hartl and A. Smits, Scaling of a small scale burner fire whirl, Combust. Flame 163, 202 (2016).

[11] A. Tohidi, M. J. Gollner, and H. Xiao, Fire Whirls, Annu. Rev. Fluid Mech. 50, 187 (2018).

[12] K. H. Chuah, K. Kuwana, and K. Saito, Modeling a fire whirl generated over a 5-cm-diameter methanol pool fire, Combust. Flame 156, 1828 (2009).

[13] W. Coenen, E. J. Kolb, A. L. Sánchez, and F. A. Williams, Observed dependence of characteristics of liquid-pool fires on swirl magnitude, Combust. Flame 205, 1 (2019).

[14] S. Leibovich, The Structure of Vortex Breakdown, Annu. Rev. Fluid Mech. 10, 221 (1978).

[15] J. H. Faler and S. Leibovich, Disrupted states of vortex flow and vortex breakdown, Phys. Fluids 20, 1385 (1977).

[16] T. Sarpkaya, On stationary and traveling vortex breakdowns, J. Fluid Mech. 45, 545 (1971).

[17] N. Syred and J. Beér, Combustion in swirling flows: A review, Combust. Flame 23, 143 (1974).

[18] S. Candel, D. Durox, T. Schuller, J.-F. Bourgouin, and J. P. Moeck, Dynamics of swirling flames, Annu. Rev. Fluid Mech. 46, 147 (2014).

[19] A. K. Gupta, D. G. Lilley, and N. Syred, Swirl flows (Abacus, Tunbridge Wells, Kent, England, 1984).

[20] S. B. Hariharan, E. T. Sluder, M. J. Gollner, and E. S. Oran, Thermal structure of the blue whirl, Proc. Combust. Inst. 37, 4285 (2019). 
[21] S. B. Hariharan, Y. Wang, P. M. Anderson, W. D. Kulatilaka, M. J. Gollner, and E. S. Oran, Understanding combustion in the blue whirl using optical diagnostics, in Proceedings of the 72nd Annual Meeting of the APS Division of Fluid Dynamics, Seattle, WA, 2019 (American Physical Society, Washington, DC, 2019).

[22] J. Chung, X. Zhang, C. Kaplan, and E. S. Oran, Blue whirl structure revealed, in Proceedings of the 72nd Annual Meeting of the APS Division of Fluid Dynamics, Seattle, WA, 2019 (American Physical Society, Washington, DC, 2019).

[23] J. Lei, N. Liu, L. Zhang, H. Chen, L. Shu, P. Chen, Z. Deng, J. Zhu, K. Satoh, and J. L. De Ris, Experimental research on combustion dynamics of medium-scale fire whirl, Proc. Combust. Inst. 33, 2407 (2011).

[24] M. D. Abràmoff, P. J. Magalhães, and S. J. Ram, Image processing with ImageJ, Biophotonics Int. 11, 36 (2004).

[25] N. Otsu, A threshold selection method from gray-level histograms, IEEE Trans. Syst., Man, Cybern. 9, 62 (1979).

[26] G. Heskestad, Luminous heights of turbulent diffusion flames, Fire Saf. J. 5, 103 (1983).

[27] B. R. Munson, D. F. Young, T. H. Okiishi, and W. W. Huebsch, Fundamentals of Fluid Mechanics, 6th ed. (Wiley \& Sons, New York, 2010).

[28] K. H. Chuah, K. Kuwana, K. Saito, and F. A. Williams, Inclined fire whirls, Proc. Combust. Inst. 33, 2417 (2011).

[29] A. Linan and F. A. Williams, Fundamental Aspects of Combustion (Oxford University, New York, 1993).

[30] H. Emmons, Fundamental problems of the free burning fire, Symp. (Int.) Combust. 10, 951 (1965).

[31] J. Lei and N. Liu, Reciprocal transitions between buoyant diffusion flame and fire whirl, Combust. Flame 167, 463 (2016).

[32] E. E. Zukoski, Properties of fire plumes, in Combustion Fundamentals of Fire (Academic, San Diego, 1995), pp. 101-219.

[33] J. G. Quintiere, Fundamentals of Fire Phenomena (John Wiley \& Sons, Chichester, UK, 2006), p. 439.

[34] J. Lei, N. Liu, L. Zhang, and K. Satoh, Temperature, velocity and air entrainment of fire whirl plume: A comprehensive experimental investigation, Combust. Flame 162, 745 (2015).

[35] S. Hariharan, Y. Hu, H. Xiao, M. Gollner, and E. S. Oran, The structure of the blue whirl, in Proceedings of the 70th Annual Meeting of the APS Division of Fluid Dynamics, Denver, Colorado, 2017 (American Physical Society, Washington, DC, 2017).

[36] A. Y. Klimenko and F. A. Williams, On the flame length in firewhirls with strong vorticity, Combust. Flame 160, 335 (2013). 